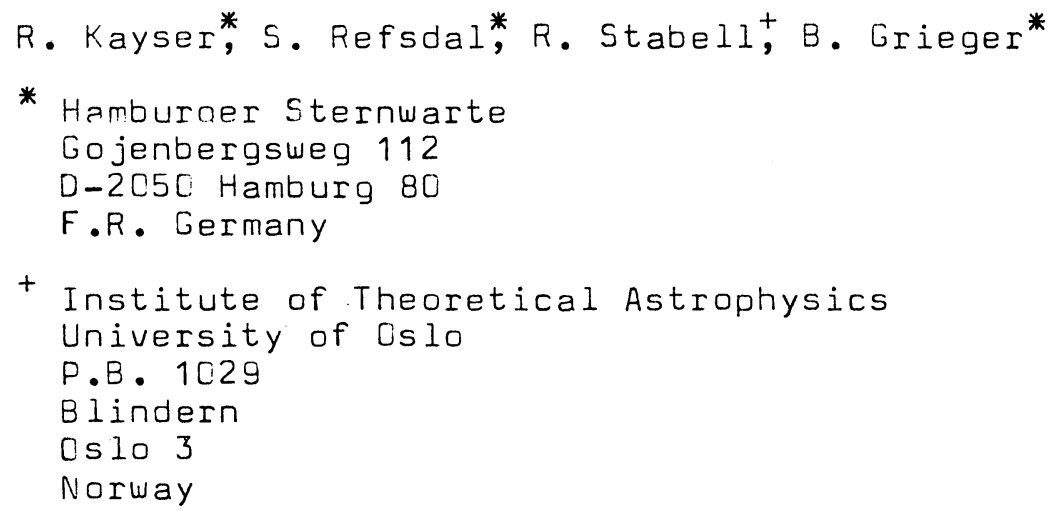

ABSTRACT. Gravitational micro-lensing due to stars in the deflecting galaxy influences the brightness and the spectra of the macro-images. Thus differences in the spectra of different macro-images are not automatically an argument against gravitational lensing. Furthermore changes in the spectra due to micro-lensing may give informations on the quasar structure. From high amplification events the brightness profile of the source may be obtained. The time scale of the high amplification event is proportional to the source radius and inverse proportional to the transversal velocity. Due to the large brightness gradient by a high amplification event, a "parallax-effect" occurs, from which the transversal velocity may be obtained, and thereby the source radius $\left(R=\Delta t \cdot V_{T}\right)$. We roughly estimate 0.3 high amplication events per year for all gravitationally lensed quasars. Frequent monitoring should be carried out in order to predict high amplification events as early as possible.

\title{
1. INTRODUCTICN
}

Gravitational micro-lensing (GMiL) due to stars in an intervening galaxy (macro-lens) may lead to a split-up of the macro-images into several micro-images.

Althpugh the separation of this micro-images is of the order $10^{-6}$ arcsec (unobservable), observable effects occur. 
Changes in brightness and in the spectra of the macroimages may lead to important informations on quasars.

\section{SPECTRAL EFFECTS}

\subsection{Chromatic Effect}

For an extended source the mean amplification 〈A> must be calculated by integration over the source. If the source radius depends on the wavelength, $\langle A\rangle$ depends on the wavelength, too.

Thus, the effect of GML is stronger for compact sources (e.g. quasar continuum source) than for more 2 extended sources (e.g. quasar emission line regions).

2.2. Equivalent width

Since the source radius is different for continuum and emission lines, $\langle A\rangle$ is also different and GML can ${ }_{2}$ therefore change the equivalent width of the emission lines.

\subsection{Emission Line Profiles}

If the emission line profile varies over the source (e.g. due to rotation) and if $A$ varies over the source, different parts of the profile are differently amplified by GML, resulting in gifferent emission line profiles for the macro-images.

\section{HIGH AMPLIF ICATION EVENTS}

\subsection{Critical curves}

The curves in the source plane where $A \rightarrow \infty$ are called critical curves. If a compact source crosses a critical curve, two micro-images appear or disappear and a typical asymmetric peak occurs in the light curve (High Amplification Event (HAE)).

\subsection{Brightness Profile and Source Radius}

By comparison of an observed light curve with the theoretical light curve of a $H A E$, the brightness profile of the soyrce (perpendicular to the critical curve) can be obtained. Especially, multiple soyrces may show up as multiple peaks in the light curve.

Since the HAE are eclipse-like events, ${ }_{2}$ their time scale is proportional to the source radius.? 
3.3. Timescale of $H A E$

The time sgale of a HAE for a typical lensed quasar (source radius $10^{-4} \mathrm{pc}$, transversal velocity $V_{T}=1000 \mathrm{~km} / \mathrm{s}$ ) is of the order 0.1 yr. For more extrem cases (e.9, 2237 +0305) time scales as short as 0.01 yr are possible.

3.4. Brightness Gradient

The steepest slope in the light curve is typically of the order $1 \mathrm{mag} /$ week ( $1 \mathrm{mag} / \mathrm{day}$ for extrem cases). This slope is due to a spatial brightness gradient at the observer: $0.25 \mathrm{mag} / \mathrm{A} . U$. for a typical lenseg quasar, $2.5 \mathrm{mag} / \mathrm{A} . U$. for extrem cases like $2237+0305$.

This gradient is easily observable by "space telescopes" $\geqslant 0.1 \mathrm{~A} . U$. away from the earth. From the time lag between the light curves measured by three observers the transversal velocity ${ }_{5}$ perpendicular to the critical curve can be obtained . Now the source radius can be obtained from the time scale of the $H A E\left(R=\Delta t \cdot V_{T}\right)$. For very small velocities the annual motion of the earth may show up as oscillations in the light curve.

3.5. Frequency of $H A E$ and Monitoring

We roughly estimate $0.1 \mathrm{HAE} / \mathrm{yr}$ for $2237+0305$ and $0.3 \mathrm{HAE} / \mathrm{yr}$ for all known gravitationally lensed quasars.

In order to predict a HAE in time, we propose a

frequent monitoring of lensed quasars. The time interval should be less than one third of the time scale of a $H A E$, i.e. about 10 days for a typical lensed quasar, an 2 days for $2237+0305$.

4. OBSERVATIONAL EVIDENCE FOR GML

If a lensed quasar shows intrinsic variations only on time scales much larger than the time delay between two images, the brightness ratio between this images would, without micro-lensing, be fairly constant. Thus, if changes of the brightness ratio are observed, they would be a clear evidence for micro-lensing in one (or both) macrorimages. $A$ variation of this kind has already been reported, for the components $A_{1}$ and $A_{2}$ of the multiple quasar $1115+080$ (separation 0.5", estimated time delay a few days). This gives evidence for GML, although further observations are needed to exclude intrinsic short-time variability of the ob ject. 
ACKNOWLEDGEMENT. This work is supported in part by the Deutsche Forschungsgemeinschaft under $A z$. Re $439 / 3$.

\section{REFERENCES}

1. K. Chang, S. Refsdal: 1979, Nature 282, 561

2. R. Kayser, S. Refsdal, R. Stabel1: $\frac{282}{1986,}$ Astron.Astrophys. 166,36

3. B. Grieger, R. Kayser, S. Refsdal: 1987, in preparation

4. K. Chang, S. Refsdal: 1984, Astron.Astrophys. 132, 168

5. B. Grieger, R. Kayser, S. Refsdal: 1986, Nature, in press

6. R. Foy, D. Bonneau, A. Blazit: 1985, Astron.Astrophys. 149, L13

7. C. Christian, D. Crabtree, P. Wadell: 1986, IAU Circular No. 4182

\section{DISCUSSION}

TURNER: In complementary work, my Princeton colleague B. Paczyns 1 ; has carried out Monte Carlo simulations of microlensing which often show substantial step function-like changes in the amplification. If microlensing affects continum and emission line regions differently, this could easily produce large spectral differences between two macro lens images. 